\title{
Tuning the Performance of I/O-Intensive Parallel Applications *
}

\author{
Anurag Acharya $a^{\ddagger \dagger} \quad$ Mustafa Uysal $1^{\ddagger} \quad$ Robert Bennett ${ }^{\ddagger} \quad$ Assaf Mendelson ${ }^{\ddagger}$ Michael Beynon ${ }^{\ddagger}$ \\ Jeff Hollingsworth ${ }^{\ddagger}$ Joel Saltz ${ }^{\ddagger \dagger}$ Alan Sussman ${ }^{\ddagger \dagger}$ \\ $\ddagger$ Dept of Computer Science \\ University of Maryland, College Park MD 20742 \\ † Center for Excellence in Space Data and Information Sciences \\ Goddard Space Flight Center, Greenbelt MD 20771
}

\begin{abstract}
Getting good I/O performance from parallel programs is a critical problem for many application domains. In this paper, we report our experience tuning the $\mathrm{I} / \mathrm{O}$ performance of four application programs from the areas of satellite-data processing and linear algebra. After tuning, three of the four applications achieve application-level I/O rates of over $100 \mathrm{MB} / \mathrm{s}$ on 16 processors. The total volume of $\mathrm{I} / \mathrm{O} \mathrm{re}-$ quired by the programs ranged from about $75 \mathrm{MB}$ to over $200 \mathrm{~GB}$. We report the lessons learned in achieving high $\mathrm{I} / \mathrm{O}$ performance from these applications, including the need for code restructuring, local disks on every node and knowledge of future $\mathrm{I} / \mathrm{O}$ requests. We also report our experience on achieving high performance on peer-to-peer configurations. Finally, we comment on the necessity of complex I/O interfaces like collective $\mathrm{I} / \mathrm{O}$ and strided requests to achieve high performance.
\end{abstract}

\section{Introduction}

$\mathrm{I} / \mathrm{O}$ has been identified as one of the major obstacles to achieving high performance from parallel computers. As a result, significant effort has been put into trying to improve the performance of $\mathrm{I} / \mathrm{O}$ on these machines. To date, most researchers have focused on observing the $\mathrm{I} / \mathrm{O}$ behavior of existing applications and on trying to improve the ability of $\mathrm{I} / \mathrm{O}$ systems available on parallel machines to execute these applications $[2,3,4,7,9]$. We take a different approach. Instead of assuming that the applications are fixed and that the $\mathrm{I} / \mathrm{O}$ system alone is open to modification, we believe that both the applications and the $\mathrm{I} / \mathrm{O}$ system have to be tuned to achieve good performance. In this paper, we concentrate on tuning the applications to improve their $\mathrm{I} / \mathrm{O}$

${ }^{*}$ This research was supported by ARPA under contract No. DABT63-94-C-0049, Caltech subcontract \#9503, by NASA under contract No. NAS5-32337, USRA/CESDIS subcontract \#555541 and by NSF under grant No. ASC9318183. Hollingsworth was supported in part by a UMCP General Research Board award.

To appear in the Fourth Annual Workshop on I/O in Parallel and Distributed Systems, May 27, 1996, Philadelphia performance, hopefully also improving their execution time. Our goal in this research was to find out what strategies were required to achieve good $\mathrm{I} / \mathrm{O}$ performance for these applications, and to identify common strategies that work for a variety of applications. We were also interested in seeing what support from I/O libraries and filesystems was necessary to achieve good performance.

To eliminate an under-configured $\mathrm{I} / \mathrm{O}$ system, often a cause of $\mathrm{I} / \mathrm{O}$ bottlenecks, as a cause of poor performance, we conducted our experiments on a parallel machine with an aggressive $\mathrm{I} / \mathrm{O}$ configuration. Our experimental platform consisted of a 16-processor IBM SP-2 with six fast disks attached to every processor. A widely used microbenchmark indicated the maximum application-level bandwidth to be $400 \mathrm{MB} / \mathrm{s}$ using the Unix raw disk interface and $270 \mathrm{MB} / \mathrm{s}$ using the Unix filesystem interface. All processors are connected to a high performance switch by a $40 \mathrm{MB} / \mathrm{s}$ bi-directional link. Each node on our platform has substantial resources and can perform $\mathrm{I} / \mathrm{O}$ for itself and for other nodes. This configuration also allowed us to investigate the performance of applications on both peer-peer and client-server architectures.

For our study, we selected I/O-intensive applications from two areas: satellite-data processing (earth science) and outof-core sparse-matrix factorization (scientific computation). The earth-science applications are currently in production use at NASA Goddard Space Flight Center and the out-ofcore sparse-matrix factorization applications have been developed at the University of Maryland with a near-term goal of solving very large submarine structural acoustics problems. I/O is required in these applications for accessing preexisting data, for intermediate results (i.e., for out-of-core processing) and for producing final output.

For each program the objective was simple: make it run as fast as possible and keep track of what was required to achieve this. The results of this exercise are encouraging. Foremost, we were able to obtain application-level I/O rates of over $100 \mathrm{MB} / \mathrm{s}$ for three out of four applications. We also observed several common characteristics in the ways in which we achieved high performance from our applications. First, although it appeared that the initial versions of some of the applications would benefit from complex I/O interfaces, such as strided requests, after tuning relatively simple I/O primitives proved to be sufficient. Second, local disks on compute nodes were required to achieve good performance for all of the applications. Third, information about future $\mathrm{I} / \mathrm{O}$ requests was available for all applications and could be 
used to schedule the requests. Both prefetching, initiated by applications, and write-behind, provided by the operating system, were successfully used.

The rest of this paper is divided into six sections. In the next section, we describe our experimental configuration. Section 3 reports on our efforts to characterize our configuration using micro-benchmarks. In Sections 4 and 5 we describe each of our application areas, report the I/O performance, and discuss the steps required to achieve this performance. Section 6 describes the lessons we learned tuning the applications. Finally, Section 7 summarizes our work.

\section{Systems background}

All our experiments were performed on a 16-node IBM SP2 running AIX 3.2.5. Each node is identically configured with one POWER2 processor, $64 \mathrm{MB}$ of main memory, two fast-wide SCSI buses and a $40 \mathrm{MB} / \mathrm{s}$ bi-directional link to a multi-stage high-performance switch. Each SCSI bus has three 2.2 GB IBM Starfire 7200 SCSI disks. The peak bandwidth for each disk is $8 \mathrm{MB} / \mathrm{s}$ and the peak bandwidth for each SCSI bus is $20 \mathrm{MB} / \mathrm{s}$. The overall system has 96 disks totaling over $200 \mathrm{~GB}$, a peak aggregate disk bandwidth of $640 \mathrm{MB} / \mathrm{s}$ and a peak aggregate SCSI bandwidth of $480 \mathrm{MB} / \mathrm{s}$. The SCSI buses and the network adaptor are connected to an $80 \mathrm{MB} / \mathrm{s}$ MicroChannel bus.

Each disk contains a separate filesystem. Although, AIX 4.1 is able to stripe filesystems across multiple disks in a single node, it has only recently become available for the SP-2 and has not yet been installed on our machine.

Jovian-2 is a multi-threaded parallel-I/O library developed at the University of Maryland. It provides an interface similar to the POSIX lio_listio() interface, which allows multiple $\mathrm{I} / \mathrm{O}$ requests to be issued with a single call. Jovian2 consists of two parts; the client proxy, which runs in the same thread as the application, and a separate server thread. The server thread can serve requests from both local and remote processes; local requests are handled as a special-case for fast processing. Jovian-2 is able to take advantage of multi-disk configurations. It allows the application process running on each node to control the scheduling of the associated I/O server. Our current implementation assumes that a standard Unix file system with asynchronous $\mathrm{I} / \mathrm{O}$ calls is available on individual nodes. On the SP-2, our implementation uses the user-space communication primitives provided by IBM's Message Passing Library (MPL).

Jovian- 2 is significantly different from the original Jovian I/O library [1]. These changes were prompted by our experience with real I/O-intensive applications and by changes in our experimental platform. There are four main differences. First, Jovian assumed a loosely synchronous model of computation and provided a collective-I/O interface; Jovian-2 makes no such assumption and provides a simple lio_listio()-like interface. Since all clients were guaranteed to participate in a request, Jovian servers would wait for requests from all their clients before issuing disk requests. Jovian-2 servers, on the other hand, try to keep the disk busy by issuing disk requests as soon as possible. Second, Jovian assumed that individual requests would be small and provided support for coalescing multiple requests. Jovian2 assumes programmers are aware of the costs of $\mathrm{I} / \mathrm{O}$ and individual $\mathrm{I} / \mathrm{O}$ requests are of substantial size. Third, Jovian servers were implemented as separate processes; Jovian2 servers are implemented as threads in the application's

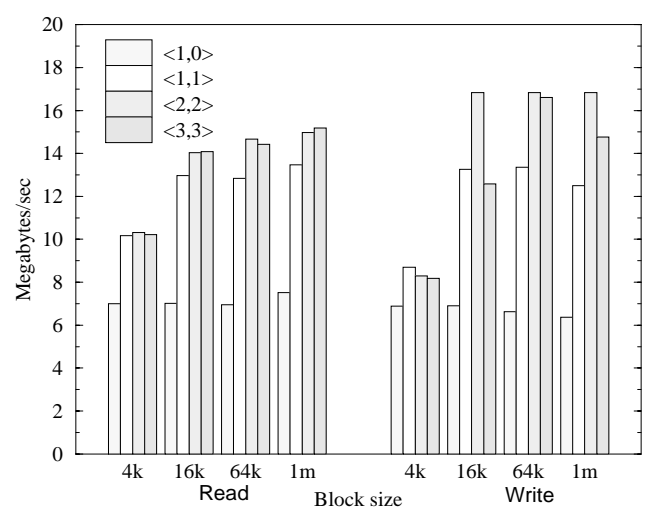

Figure 1: Maximum application-level I/O rates for JFS. $\langle x, y\rangle$ indicates the number of disks on each of the two SCSI buses.

address-space. Finally, Jovian did not provide support for striping over multiple disks whereas Jovian-2 supports usercustomizable striping.

\section{Micro-benchmarks}

We conducted a series of experiments using micro-benchmarks to characterize the performance of our experimental platform. These experiments had two goals. First, we wanted to determine the maximum application-level I/O bandwidth, which is a more realistic baseline to evaluate the performance of applications than peak disk or SCSI transfer rates. Second, we wanted to determine the parameters and configurations that provide the best performance. This information is needed to tune applications to effectively use the $\mathrm{I} / \mathrm{O}$ system.

In this section we present results for both the native Unix filesystem available on the SP-2 and for Jovian-2. For our study, we used Jovian- 2 for the earth-science applications and the Unix filesystem for the sparse-matrix factorization applications.

\subsection{Journaled File System}

The Journaled File System is the default Unix filesystem available on AIX 3.2. To measure single node JFS performance, we used a modified version of the widely used iozone benchmark [13]. iozone determines the maximum application-level $\mathrm{I} / \mathrm{O}$ bandwidth by making a sequence of contiguous write requests followed by a sequence of contiguous read requests. Our version of this program supports multi-disk configurations and can generate multiple simultaneous requests per disk. It issues all requests using a single lio_listio() call and waits for all of them to complete before issuing the next set.

We performed experiments for request sizes between $4 \mathrm{~KB}$ and $4 \mathrm{MB}$; with one to six outstanding requests per disk and six different disk configurations. For each experiment, the benchmark wrote and read back a $70 \mathrm{MB}$ file. We used a $70 \mathrm{MB}$ file to ensure we measured disk activity and not file cache performance. Along the request-size dimension, the bandwidth curves saturate around $1 \mathrm{MB}$ requests. Increasing the number of outstanding requests did not provide much 
benefit. In most cases, the best performance was achieved with just one or two outstanding requests. Of the various configurations tested, the configuration with two disks per SCSI bus provided the best performance in almost all cases. The additional bandwidth provided by adding a third disk on a SCSI bus did not increase performance because of contention between disks for the bus.

Figure 1 presents the maximum application-level read and write bandwidths for a set of request sizes on four disk configurations. We repeated these experiments using the Unix raw disk interface instead of JFS. The maximum read bandwidth achieved was $25.2 \mathrm{MB} / \mathrm{s}$ and the maximum write bandwidth was $23.5 \mathrm{MB} / \mathrm{s}$. Although the raw disk configuration provided noticeably higher throughput, we decided that any potential benefit from using raw disks would be offset by the loss in functionality.

\subsection{Jovian-2}

To test the performance of the Jovian-2 parallel-I/O library for various $\mathrm{I} / \mathrm{O}$ configurations, we performed experiments for three kinds of configurations:

- Local-access: data is located on the same node that the $\mathrm{I} / \mathrm{O}$ requests are made on.

- Client-server: the nodes participating in the experiment are partitioned into clients and servers as shown in Figure 2(a). Clients run both the application thread and the $\mathrm{I} / \mathrm{O}$-server thread whereas servers run only the $\mathrm{I} / \mathrm{O}$-server thread. All I/O requests are made on clients.

- Peer-peer: all nodes run both the application and the $\mathrm{I} / \mathrm{O}$-server thread as shown in Figure 2(b). I/O requests can originate from any node.

In these experiments, the primary parameter was the number of nodes that performed I/O. On each node that performed I/O, we used four disks (two on each SCSI bus), since that provided the best performance, as shown in Section 3.1. Both file size and request size were scaled with the number of disks. For the local-access and the one-client-oneserver cases, a single processor wrote and read back a $70 \mathrm{MB}$ file. For larger configurations, the file size was scaled to ensure at least $70 \mathrm{MB}$ of data per node. file In addition, we read a $70 \mathrm{MB}$ file on every node between every write experiment and the subsequent read experiment. For each configuration, the file was striped over all available disks - local disks for the local-access case, server disks for client-server configurations, and all disks on all the nodes for peer-peer configurations.

The request size was scaled to request at least one striping unit from every disk. For a configuration that used $d$ disks, a request of striping_unit_size $\times d \mathrm{~KB}$ was used. For configurations with multiple clients (or multiple peers), non-overlapping requests were generated. For peer-peer configurations using $n$ nodes, $1 / n$ of $\mathrm{I} / \mathrm{O}$ was local and the rest remote.

Results for a representative subset of the experiments are presented in Table 1. All measurements include time required to flush the file cache. For comparison, the maximum JFS bandwidths for four disks per node are $15.0 \mathrm{MB} / \mathrm{s}$ (read) and $16.8 \mathrm{MB} / \mathrm{s}$ (write). For non-local requests, Jovian-2 reads files from disk using JFS and delivers them to the requesting application using MPL. An upper bound for the bandwidth that can be achieved for individual non-local requests can be computed by adding the time taken to read a striping unit from the disk into the memory of the server node and the time taken to move it to the client node. We measured the maximum communication bandwidth for $128 \mathrm{~KB}$ messages to be $32 \mathrm{MB} / \mathrm{s}^{1}$ Combining this with the maximum application-level I/O bandwidth via JFS (from Table 1), we arrive at $10.3 \mathrm{MB} / \mathrm{s}$ as an upper bound for non-local bandwidth for individual requests. In comparison, Jovian- 2 achieved a read bandwidth of $9.3 \mathrm{MB} / \mathrm{s}$ for a one-server-one-client configuration. For configurations with relatively large $\mathrm{I} / \mathrm{O}$ bandwidth - more servers than clients and large peer-peer systems, the read and write bandwidths are comparable. For configurations with relatively small I/O bandwidth, write bandwidth is much higher than read bandwidth, due to write-behind in the filesystem.

\section{Earth-Science Applications}

The two earth-science programs, included in our application suite, pathfinder and climate, constitute a processing chain for Advanced Very High Resolution Radiometer (AVHRR) data. The Pathfinder AVHRR data sets are global, multichannel data from NOAA meteorological satellites. Both pathfinder and climate are currently in production use by NASA's Goddard Distributed Active Archive Center and, together, are representative of a large class of NASA earthscience applications. Furthermore, the structure of these applications is similar to the large set of programs currently being developed to process data from the Earth Observation System [6] satellites.

Pathfinder: This program is the first in the processing chain and processes AVHRR global area coverage data. Its input consists one or more daily data sets which contain satellite imagery and several ancillary files which contain topographic and cartographic data about the earth and information that helps determine the position of the satellite at any given time. Each daily data set contains fourteen files, each containing data corresponding to a little more than one orbit. The output of the program is a single multichannel image of the world. Pathfinder performs calibration for instrument drift, topographic correction, masking of cloudy pixels, registration of individual pixels with locations on the ground and compositing of multiple pixels corresponding to the same ground location. The size of one daily data set is about $680 \mathrm{MB}$, the total size of all ancillary files is about $100 \mathrm{MB}$ and the size of the output is $228 \mathrm{MB}$.

Each orbit file consists of about 10,000-13,000 scan lines, each scan line containing 3584 bytes. Input data is read in scan line by scan line, in chunks of 128 lines. Processing is done one chunk at a time. Ancillary files are not cached in memory. Instead, the data from these files is read when needed. The calibration, correction, masking and registration operations depend only on the data in the chunk being processed; the composition operation combines data from multiple chunks. For the first four operations, pathfinder maintains large in-core scratch data-structures which are reused for every chunk. For composition, pathfinder maintains an out-of-core intermediate version of the composite image. After the first four operations have been performed on a chunk, each data value in the chunk is mapped into the

\footnotetext{
${ }^{1}$ This measurement was done by determining the time required to send one million $128 \mathrm{~KB}$ messages between a pair of nodes.
} 


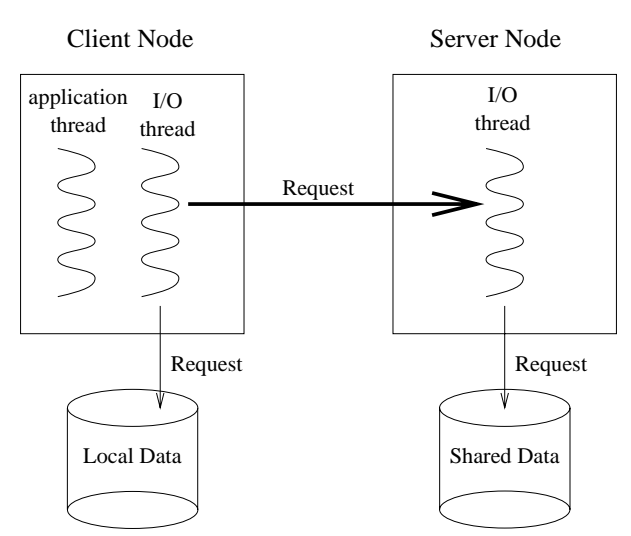

(a)

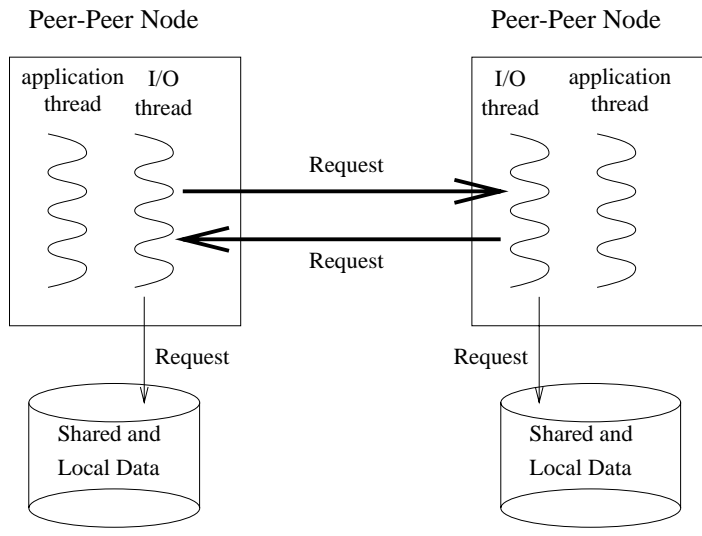

(b)

Figure 2: Configurations for (a) client-server and (b) peer-peer execution models

Four-way striping on each node (2 disks per SCSI bus), $128 \mathrm{~KB}$ striping unit

\begin{tabular}{|c|c|c|c|c|c|c|c|c|c|c|c|c|c|}
\hline \multirow[b]{2}{*}{ Configuration } & & \multicolumn{4}{|c|}{ one client,varying servers } & 4 nodes & \multicolumn{4}{|c|}{8 nodes } & \multicolumn{3}{|c|}{ Peer-peer } \\
\hline & local & c1-s1 & c1-s2 & c1-s4 & c1-s8 & C3-s1 & $c 7-s 1$ & $c 6-82$ & c5-s3 & c4-s4 & pp-2 & pp-4 & $\mathrm{pp}-8$ \\
\hline Read bandwidth & 14.3 & 9.3 & 17.5 & 21.2 & 22.8 & 8.5 & 8.9 & 16.6 & 23.3 & 25.9 & 13.8 & 16.5 & 26.0 \\
\hline Write bandwidth & 16.7 & 8.7 & 16.3 & 20.6 & 21.6 & 9.7 & 19.8 & 24.8 & 30.0 & 27.9 & 21.2 & 26.2 & 33.2 \\
\hline
\end{tabular}

Table 1: Micro-benchmark performance for Jovian-2. All bandwidths are aggregate and are in MB/s. cx-sy corresponds to a client-server configuration with $x$ clients and $y$ servers; pp- $x$ corresponds to a peer-peer configuration with $x$ peers.

intermediate image and is compared with the corresponding pixel. If the new value is "better", it is copied into the pixel. In effect, the composition operation has been implemented as an out-of-core max-reduction. Note that the mapping between the pixels in the satellite images and the two-dimensional output image generated by pathfinder is complex and many-to-one. Once all input data has been processed, the intermediate image is scanned for pixels that have no data associated with them. This happens, for example, if the satellite image is clouded. Data for such pixels is computed by interpolation. Finally, the output image is generated by concatenating information about the data set with the intermediate image. The total I/O performed by an optimized sequential version, including $\mathrm{I} / \mathrm{O}$ for out-of-core accesses, is over $28 \mathrm{~GB}$.

Although processing of the AVHRR global area coverage data is representative of many earth science applications, some similar programs process even more data. For example, the input volume is sixteen times higher for the AVHRR local area coverage data which is higher resolution; the corresponding output size for the global $1 \mathrm{~km}$ data products is sixty-four times larger. For MODIS, the primary instrument of the Earth Observation System, both the input and the output are at least two orders of magnitude larger than the AVHRR global area coverage data processed by pathfinder.

Climate: This program processes the output of pathf inder and generates the AVHRR Land Climate data product. It performs a data selection and reduction operation. It extracts three frequency bands of the image generated by pathfinder and reduces them to a single band latitudelongitude grid. The output of climate is vegetation index map which is used to track global land cover change. Input to climate is the $228 \mathrm{MB}$ file generated by pathfinder, of which the program reads $54 \mathrm{MB}$. In addition, climate reads $21.5 \mathrm{MB}$ from an ancillary file. The output image is about $130 \mathrm{~KB}$. The total $\mathrm{I} / \mathrm{O}$ volume for climate is $75.5 \mathrm{MB}$.

\section{I/O Optimizations:}

- In both programs, input was being read one scan line at a time (3.5KB for pathfinder, $10 \mathrm{~KB}$ for climate). We aggregated input reads to $512 \mathrm{~KB}$ in both cases.

- A recurrent I/O pattern in both programs was the embedding of small $\mathrm{I} / \mathrm{O}$ requests in the innermost loops. Each such occurrence generated nested sequences of small requests with fixed strides. This occurs in three situations: (1) reading of topographic data, (2) reading of the land-sea mask and (3) reading and writing of dat a for the out-of-core max-reduction. The request size was almost always two bytes and the subsequent seek distance was 20 MB. Relatively straightforward loop restructuring transformations were sufficient to aggregate the $\mathrm{I} / \mathrm{O}$ and move it to the outermost loop. In the first two cases, the $\mathrm{I} / \mathrm{O}$ was converted to block reads, whereas for the out-of-core max-reduction, it was converted to block read-modify-writes.

- All I/O in both programs was buffered, using the stdio library with $4 \mathrm{~KB}$ buffers. In most cases, including the patterns described above, this buffering was inappropriate. In the case of the patterns described above, individual requests were small (two bytes) and the distance between successive accesses (20 MB) was very 
large. We replaced the buffered-I/O calls by their unbuffered analogues.

\section{Parallelization:}

In pathfinder, the map between the input satellite images and the output global composite image is data-dependent and cannot be computed a-priori. The amount of computation depends roughly upon the size of the input data processed. However, this relationship is weak as: (1) night images are not processed, (2) clouded images are partially processed and (3) ocean images are partially processed. The categorization of an image is also data-dependent and can be determined only after the data has been unpacked and partially processed.

We parallelized pathfinder by partitioning the output image in equal-sized horizontal strips. Each processor is responsible for all processing needed to generate its partition of the output image. We chose to partition the output image (instead of the input data) as this allows all combination operations $^{2}$ to be local to individual processors. No interprocessor communication is needed. We chose a horizontal partitioning scheme to take advantage of the row-major storage format used in all files (input, ancillary as well as output files). Horizontal striping allows $\mathrm{I} / \mathrm{O}$ to be performed in large contiguous blocks.

Each processor computes the map from the input data set to the output image by subsampling (one scan line per chunk) all input files. It then reads the chunks that intersect with its partition of the output image. For each chunk, it maps each input pixel into the output image. Pixels that map into its partition are processed further, others are ignored. The individual partitions of the output image are also too large to be stored in main memory. Therefore, the composition operation is still out-of-core. Once all processing is completed, the final result is produced by concatenating the individual partitions.

In climate, the mapping between the pixels of the input image and those of the output image is data-independent and can be computed a-priori. The amount of computation to be done is proportional to the amount of input data. We parallelized climate by horizontally partitioning the output image. Each processor reads the data that maps to its partition of the output image. Load balance is achieved by ensuring that all processors read approximately equal amounts of data.

For both pathf inder and climate, the final image is generated by concatenating the images generated by individual processors.

\section{Use of Parallel I/O:}

In our experiments, we used four disks per node, two disks on each SCSI bus. We replaced calls to Unix I/O routines by calls to Jovian- 2 routines. All ancillary files were replicated and striped across the four disks on every node. For client-server configurations, all input and output files were striped over all the disks of all server nodes; for peer-peer configurations, these files were striped over all participating disks. Every node running a pathfinder process (that is, the clients in client-server configurations and all participating nodes in peer-peer configurations) created

${ }^{2}$ All input pixels that map to a single pixel in the output image are combined by a max-reduction operations to get the final value of an output pixel. a separate temporary file to hold its partition of the intermediate image. This file was striped over the four local disks. The striping-unit size in all cases was $128 \mathrm{~KB}$.

\subsection{Results and Analysis}

We ran pathfinder and climate for one daily data set on a variety of configurations. An unmodified version of pathfinder ran for 18,800 seconds on a single processor of the SP-2. Of this, about 13,600 seconds $(76 \%)$ of the time was spent waiting for $I / O ; 580$ seconds for input, 50 seconds for output and the remaining 12,970 seconds of $\mathrm{I} / \mathrm{O}$ for the out-of-core max-reduction.

Table 2 shows the breakdown of total I/O volume for the parallel version of pathfinder. The volume changes with configuration for two reasons. First, every processor constructs the map from the input images to the output image by reading one scan line per chunk of 128 scan lines. As a result, the total amount of input grows with the number of processors that are running the application (clients in client-server, all nodes in peer-peer). This growth can be avoided by partitioning the task of constructing the map between input and output coordinates and having each processor report its share of the map to all other processors. Second, the size of the block that is read during the outof-core max-reduction is determined by the bounding box around the pixels that are to be updated. Since the pixels to be updated are sparsely distributed, finer partitions of the intermediate image file are able to eliminate holes, reducing the total volume of $\mathrm{I} / \mathrm{O}$ for this phase. The volume of intermediate reads is consistently much higher than the volume of intermediate writes. This is because some on-disk data has to be read to decide whether any pixels are to be updated. Writes are needed only if at least one of the pixels need to be updated, and then only for the bounding box around the pixels to be updated.

The breakdown of total I/O volume for climate does not change with configuration. The $\mathrm{I} / \mathrm{O}$ for climate consists almost exclusively of read requests. Total local I/O (for ancillary files) is about $21.5 \mathrm{MB}$ and total non-local $\mathrm{I} / \mathrm{O}$ (input data) is $54 \mathrm{MB}$. The output volume is $130 \mathrm{~KB}$.

Early results indicated that there was a large difference between the performance of peer-peer and client-server configurations for pathfinder. With abbreviated input (one orbit file instead of fourteen), pathfinder running on a four processor peer-peer configuration was able to achieve only a $400 \mathrm{~KB} / \mathrm{s}$ per-processor non-local read bandwidth. With the same input, it was able to achieve a per-processor nonlocal read bandwidth of $6 \mathrm{MB} / \mathrm{s}$ on a client-server configuration of the same size (three clients, one server). The write bandwidth on a four-processor peer-peer configuration was better at $3 \mathrm{MB} / \mathrm{s}$ but was much lower than the $7.4 \mathrm{MB} / \mathrm{s}$ achieved on the corresponding client-server configuration of same size (three clients, one server). Note that the non-local reads are used to input the satellite data in chunks of 128 scan lines and are interspersed with computation, whereas the non-local writes are used for final output and are bunched together. The total execution time for an abbreviated pathfinder run (single orbit file) on a four processor peer-peer configuration was 510 seconds, of which 320 seconds was $\mathrm{I} / \mathrm{O}$ waiting time. In comparison, the execution time on a three-client-one-server configuration was 290 seconds, of which 103 seconds was $\mathrm{I} / \mathrm{O}$ waiting time. In contrast, the Jovian- 2 micro-benchmark, which does no computation, achieved comparable performance on 


\begin{tabular}{|c|c|c|c|c|c|c|c|c|c|c|}
\hline \multirow[b]{2}{*}{ Config (client/server) } & 4 nodes & \multicolumn{3}{|c|}{8 nodes } & \multicolumn{3}{|c|}{12 nodes } & \multicolumn{3}{|c|}{16 nodes } \\
\hline & $\mathrm{c} 3-\mathrm{s} 1$ & c7-s1 & $\mathrm{c} 6-\mathrm{s} 2$ & $\mathrm{c5-s3}$ & c11-s1 & $\mathrm{c} 10-\mathrm{s} 2$ & $\begin{array}{c}\mathrm{c} 9-\mathrm{s} 3 \\
\end{array}$ & c15-s1 & c14-s2 & c13-s3 \\
\hline Input & 1,508 & 3,100 & 2,700 & 1,838 & 4,751 & 4,334 & 3,924 & 6,350 & 5,952 & 5,561 \\
\hline Intermediate read & 20,341 & 12,306 & 13,143 & 11,261 & 10,507 & 10,699 & 11,094 & 9,275 & 9,600 & 9,893 \\
\hline Intermediate write & 6,301 & 4,493 & 4,701 & 3,697 & 3,973 & 4,039 & 4,130 & 3,683 & 3,871 & 3,809 \\
\hline Total & 28,378 & 20,126 & 20,771 & 17,024 & 19,459 & 19,300 & 19,376 & 19,536 & 19,651 & 19,492 \\
\hline
\end{tabular}

Table 2: Breakdown of total I/O volume (in MB) for pathfinder. Output volume is 228 MB for all configurations.

both peer-peer and client-server configurations (see Table 1). This might lead to speculation that applications that do significant amount of computation hamper I/O performance on peer-peer configurations. Section 5 provides a counterexample. It provides performance results for a program that performs substantial computation and $\mathrm{I} / \mathrm{O}$ on a peer-peer configuration. It achieves good performance by using extensive global information about future $\mathrm{I} / \mathrm{O}$ requirements and a one-sided communication model. Our current hypothesis is knowledge of future $\mathrm{I} / \mathrm{O}$ requirements is necessary to achieve good computation and $\mathrm{I} / \mathrm{O}$ performance on peer-peer configurations. We intend to test this hypothesis in our future research. For the rest of the experiments with pathfinder and climate, we limited ourselves to client-server configurations.

Figure 3 shows a breakdown of execution time for pathfinder for a set of client-server configurations. There are three interesting points. First, pathfinder is now compute-bound. Except for the 15-client-1-server case, I/O waiting time is less than $25 \%$ of the total time. In many cases, it is substantially less ( $10 \%$ in one case). Second, for a given number of nodes, configurations with a small number of servers achieved the best performance. This is unsurprising as the bulk of the $\mathrm{I} / \mathrm{O}$ is for intermediate read/write operations and is directed to local disks. It should be noted that increasing servers in a fixed size configuration has two conflicting effects: (1) it increases the bandwidth for nonlocal $\mathrm{I} / \mathrm{O}$ (by increasing the number of nodes that act as servers) and (2) it decreases the bandwidth for local $\mathrm{I} / \mathrm{O}$ (by reducing the number of clients). Increasing the number of servers beyond two for any of the configurations provided no benefit and actually increased the execution time for the twelve- and sixteen-processor configurations. Third, the execution time does not reduce significantly from the twelveprocessor to the sixteen-processor configurations. There are two reasons for this. First, since all processors independently compute the map from input coordinates to output coordinates, the amount of input data read during the partitioning phase increases with the number of processors. Second, as the number of processors grows, each chunk (128 input scan lines) is partitioned between more processors. Each processor that processes a part of a chunk has to unpack, parse and map the entire chunk before it is able to isolate the portion it needs to process. Therefore, the total amount of processing done on every chunk grows roughly with the number of processors. As was mentioned earlier, growth in the amount of total input read volume can be avoided by partitioning the task of computing the map between input and output coordinates, followed by a global exchange of information. An even better solution would be to compute the map from satellite image coordinates to the output image coordinates during the process of converting raw sensor read- ings (level 0 data in NASA parlance) to the AVHRR orbital data files (level $1 \mathrm{~b}$ data in NASA parlance). This conversion occurs earlier in the processing chain than pathfinder. This change would also help eliminate the growth in the total amount of computation as individual processors can now read only the portions of chunks that map to their partition of the output image. Note that this would require restructuring of pathfinder code to process variable-size chunks.

Table 3 presents the aggregate bandwidths sustained by pathfinder for different kinds of $\mathrm{I} / \mathrm{O}$. Recall that both input and output $\mathrm{I} / \mathrm{O}$ are non-local, whereas both intermediate reads and writes are to local disks. It is interesting to note that for configurations with many clients and few servers, the aggregate $\mathrm{I} / \mathrm{O}$ rate achieved is greater than the value indicated by the micro-benchmark results presented in Table 1. This is made possible by the fact that, beyond an initial barrier for configuration purposes, all client processes are independent. This allows different clients to utilize the server(s) at different times. A parallel-I/O library that provides a collective- $\mathrm{I} / \mathrm{O}$ interface and coalescing of requests from multiple clients would usually not be able to do this as it would wait until requests are received from all clients before issuing any requests to the disks. A collective-I/O library that provided only partial coalescing and issued disk requests without waiting for all requests to arrive, would be able to utilize the server(s) over a longer time.

Another point of interest is that the intermediate requests have substantial locality and are able to take good advantage of the operating-system file cache for both reuse and write-behind. This is facilitated by the parallelization scheme, which ensures that all intermediate $\mathrm{I} / \mathrm{O}$ is to local disks and that each processor processes exactly the data that maps into its segment of the output image.

Figure 4 shows the breakdown of execution time for climate. Computation for climate scales well. The total I/O time was consistently about $4-5 \%$ of the total computation time for all the configurations we experimented with. We did not run climate on larger configurations, since the individual partitions of the input data would become very small. Recall that the total $\mathrm{I} / \mathrm{O}$ volume for climate is $75.5 \mathrm{MB}$. Table 4 presents the aggregate $\mathrm{I} / \mathrm{O}$ rates for climate. As in the results for pathfinder, independent requests allow climate to achieve a larger aggregate bandwidth than was indicated by the Jovian- 2 micro-benchmark.

Table 5 presents end-to-end I/O rates for both pathfinder and climate. It shows that both programs are now compute-bound. It also shows that it is possible to achieve end-to-end $\mathrm{I} / \mathrm{O}$ rates over $26 \mathrm{MB} / \mathrm{s}$ in earth-science applications. 


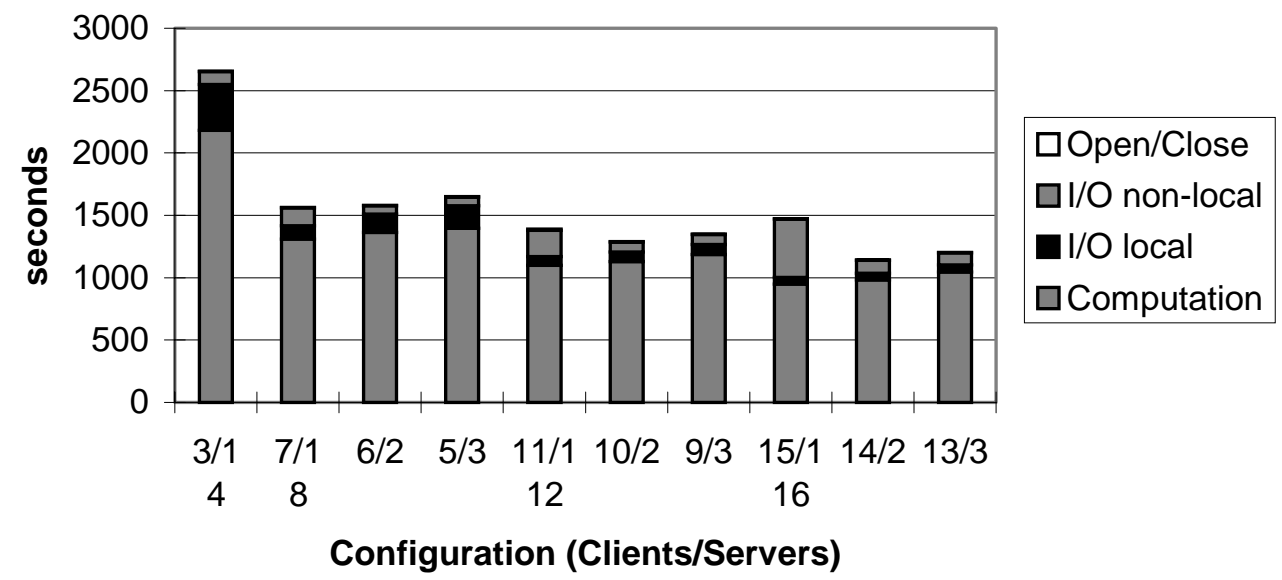

Figure 3: Breakdown of execution time for pathfinder. The numbers in the bottom row indicate the total number of nodes in the configuration. An unmodified version of pathfinder ran for 18,800 seconds on a single processor of the SP-2. Of this, about 13,600 seconds $(76 \%)$ of the time was spent waiting for read/write operations.

\begin{tabular}{|c|c|c|c|c|c|c|c|c|c|c|}
\hline \multirow[b]{2}{*}{ Config (client/server) } & 4 nodes & \multicolumn{3}{|c|}{8 nodes } & \multicolumn{3}{|c|}{12 nodes } & \multicolumn{3}{|c|}{16 nodes } \\
\hline & $\mathrm{c} 3-\mathrm{s} 1$ & $c 7-s 1$ & $c 6-s 2$ & $\mathrm{c} 5-\mathrm{s} 3$ & c11-s1 & c10-s2 & c9-s3 & c15-s1 & c14-s2 & $\mathrm{c} 13-\mathrm{s} 3$ \\
\hline Input & 18.0 & 25.4 & 45.6 & 38.5 & 26.4 & 60.0 & 60.3 & 15.0 & 65.8 & 68.9 \\
\hline Output & 22.2 & 34.3 & 55.8 & $\overline{40.0}$ & $\overline{41.8}$ & 108.0 & 94.5 & 63.0 & 126.0 & 135.2 \\
\hline Intermediate read & 85.8 & 246.4 & 192.6 & 151.5 & 510.4 & 453.0 & 388.8 & 634.5 & 644.0 & 600.6 \\
\hline Intermediate write & 65.7 & 169.4 & 147.2 & 118.0 & 278.3 & 256.0 & 230.4 & 406.5 & 372.4 & 338.0 \\
\hline Overall & 64.8 & 96.2 & 122.1 & 103.5 & 85.0 & 163.0 & 162.5 & 43.8 & 161.6 & 170.0 \\
\hline
\end{tabular}

Table 3: Aggregate application-level I/O rates for pathfinder. All rates are in MB/s. The aggregate I/O rate is computed by multiplying the per processor application-level $\mathrm{I} / \mathrm{O}$ rate by the number of clients. Per processor $\mathrm{I} / \mathrm{O}$ rate is computed as the sum of $\mathrm{I} / \mathrm{O}$ volumes for all clients divided by the sum of time spent in $\mathrm{I} / \mathrm{O}$ routines by all clients.

\begin{tabular}{|c|c|c|c|c|c|c|c|c|c|c|}
\hline \multirow[b]{2}{*}{ Config (client/server) } & 4 nodes & \multicolumn{3}{|c|}{8 nodes } & \multicolumn{3}{|c|}{12 nodes } & \multicolumn{3}{|c|}{16 nodes } \\
\hline & C3-s1 & c7-s1 & $c 6-s^{2}$ & $\mathrm{c} 5-\mathrm{s} 3$ & c11-s1 & c10-s2 & c9-s3 & c15-s1 & c14-s2 & c13-s3 \\
\hline pathfinder & 11.8 & 17.9 & 17.5 & 16.2 & 20.8 & 22.7 & 21.7 & 18.8 & 26.5 & 25.5 \\
\hline climate & 1.2 & 2.5 & 2.3 & 1.9 & - & - & - & - & - & - \\
\hline
\end{tabular}

Table 5: End-to-end I/O rates for pathfinder and climate. All rates are in $\mathrm{MB} / \mathrm{s}$. The rate is computed by dividing the total data volume by the total execution time. 


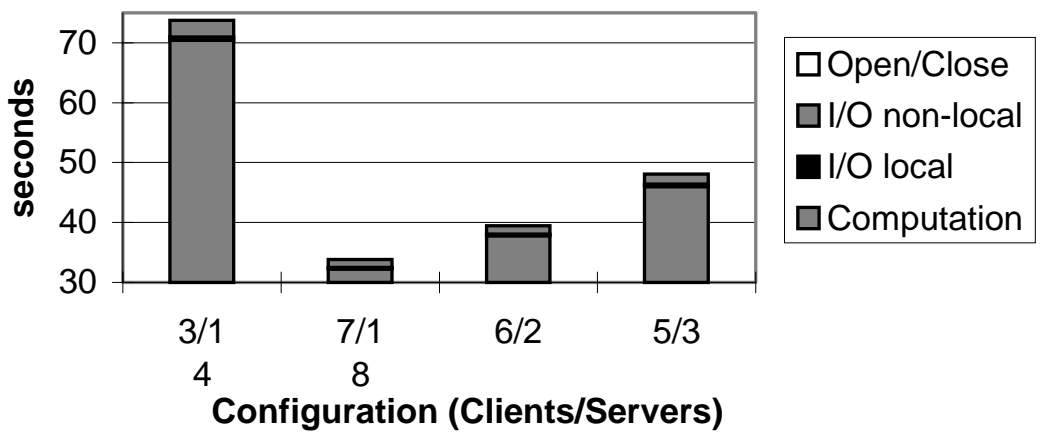

Figure 4: Breakdown of execution time for climate. The numbers in the bottom row indicate the total number of nodes in the configuration.

\begin{tabular}{|l|c||c|c|c|}
\hline \multirow{2}{*}{ Config (client/server) } & \multicolumn{1}{|c||}{4 nodes } & \multicolumn{3}{c|}{8 nodes } \\
\cline { 2 - 5 } & c3-s1 & c7-s1 & c6-s2 & c5-s3 \\
\hline \hline Local I/O & 35.1 & 77.0 & 64.2 & 56.5 \\
\hline Non-local I/O & 17.7 & 25.2 & 34.2 & 31.5 \\
\hline Overall & 19.2 & 27.8 & 36.0 & 32.7 \\
\hline
\end{tabular}

Table 4: Aggregate application-level I/O rates for climate, in $\mathrm{MB} / \mathrm{s}$. The aggregate $\mathrm{I} / \mathrm{O}$ rate is computed by multiplying the per-processor application-level $\mathrm{I} / \mathrm{O}$ rate by the number of clients. Per-processor I/O rate is computed as the sum of $\mathrm{I} / \mathrm{O}$ volumes for all clients divided by the sum of time spent in $\mathrm{I} / \mathrm{O}$ routines by all clients.

\section{Out-of-core Sparse-Matrix Factorization}

Many scientific and engineering applications require the solution of very large sparse linear systems. Assuming a total memory pool of $50 \mathrm{~GB}$, the largest sparse system (with $5 \%$ sparsity and double-precision complex arithmetic) that can be solved in-core on current supercomputers consists of about 250,000 equations. ${ }^{3}$ Demands of some applications are far beyond that limit. In particular, submarine structural acoustics problems can require the solution of sparse linear systems with 2-3 million equations. Such applications require efficient out-of-core methods. We have implemented an out-of-core parallel sparse Cholesky factorization, along with associated programs for parallel symbolic factorization and parallel matrix partitioning. We have selected two of these programs, the sparse-matrix partitioner (partitioner) and the Cholesky factorization programs (factor) for our study. Like pathf inder and climate, this pair of programs forms a processing chain.

Sparse Cholesky factorization arises in the direct solution of symmetric positive-definite systems of linear equations. The Cholesky factor of a symmetric positive definite matrix $A$ is a lower-triangular matrix $L$ with positive $\operatorname{diag}$ onal, such that $A=L L^{T}$. Our parallel out-of-core sparse Cholesky factorization is a parallelization of a left-looking supernodal Cholesky factorization algorithm [11]. This particular formulation of Cholesky factorization is based on $s u$ -

\footnotetext{
${ }^{3}$ We arrive at this number by calculating the number of doubleprecision complex values that a 50 GB memory will hold and by using this number and the sparsity to compute the corresponding number of equations. This number is an overestimate, as it ignores the memory required to hold the data-structures used to efficiently store the sparse matrix.
}

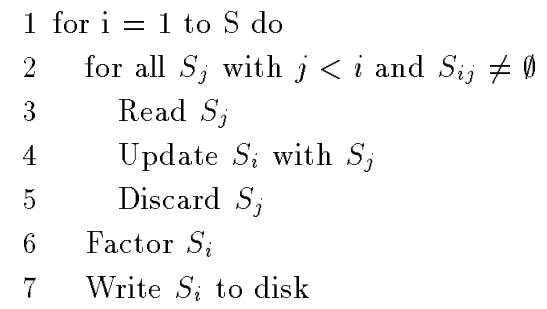

Figure 5: Out-of-core Sparse Cholesky Factorization

pernodes. Each supernode is a set of contiguous columns such that every adjacent column in the set has an identical sparsity structure below the diagonal. Using supernodes enables the use of efficient dense linear-algebra kernels [5], as well as large transfers between secondary storage and primary memory. These applications assume a peer-peer configuration and directly use Unix I/O calls.

Partitioner: this program has two input files, the matrix file which contains the structure of the original matrix $(A)$ and its non-zero values, and the index file which contains the sparsity structure of the factor $L$. The index file is generated by a symbolic factorization of $A$ prior to the execution of partitioner. Partitioner performs two operations: (1) computing and allocating space for the fill-ins, which are locations in $A$ that are originally zero, but will become nonzero (in $L$ ) after the factorization; and (2) distributing the Cholesky factor, $L$, to the processors participating in the factorization.

The Cholesky factor is partitioned using a 2-D strategy originally developed in [17]. The processors are organized in a $k \times m$ grid. Let $P_{r, q}$ denote the processor number at the $r$ th row and $q$ th column of the processor grid. Supernode $i$ of matrix $A$ is mapped to processors in the $(i \bmod m)^{t h}$ column of the processor grid. A supernode is further partitioned among the processors in a column of the processor grid, such that block $j$ of supernode $i$ is mapped to processor $P_{j} \bmod k, i \bmod m$. This mapping ensures that communication takes place only within the processors placed in the same column or in the same row of the processor grid. Hence, each processor communicates with at most $k+m$ other processors.

This program has two phases with similar I/O access 
patterns. The first phase sequentially reads the index file to extract the supernodal structure of the matrix. All requests in this phase are very large (25 MB) and contiguous. Furthermore, all requests are read requests and use blocking $\mathrm{I} / \mathrm{O}$ calls. With the exception of the columns on the partition boundaries, all $\mathrm{I} / \mathrm{O}$ in this phase is to the local disk. Requests in the second phase also access large contiguous chunks but the request size is smaller $(5 \mathrm{MB})$ and two local files, instead of one, are used.

Factor: as mentioned above, this program implements leftlooking supernodal Cholesky factorization. Figure 5 provides a high-level algorithm. Parallelism in factor is achieved at several levels, both in computation and $\mathrm{I} / \mathrm{O}$. First, since each supernode is partitioned among $k$ processors, updates to a supernode are performed in parallel. Second, multiple supernodes can be updated in parallel, as long as the dependences are satisfied. That is, supernode $S_{j}$ can update supernode $S_{i}$ as long as the factorization (step 6) has been performed on $S_{j}$. In our implementation, at most $m$ supernodes are updated in parallel, where $m$ is the horizontal dimension used in the processor grid. Third, each supernode is striped across $k$ processors. The stripe size depends on the sparsity of the supernode and is determined by partitioner. Fourth, asynchronous I/O primitives are used to overlap the computation with $\mathrm{I} / \mathrm{O}$. The prefetch mechanism uses a pre-computed schedule to issue as many asynchronous $\mathrm{I} / \mathrm{O}$ requests as possible given the memory constraints. The requests are issued in the order that the corresponding data will be used. We have not attempted to improve the communication balance for the factor. Our relatively simple technique provided acceptable performance for moderately unstructured matrices but did not perform well on sara-2, which is very sparse.

A key data structure in factor is the elimination tree [10] generated during symbolic factorization using the structure of the sparse matrix. This structure contains dependency information between different supernodes and does not change over the course of the computation. Therefore the exact sequence of supernode update operations is known a-priori and can be used to generate a schedule for $\mathrm{I} / \mathrm{O}$ and communication for all processors. This information can be exploited when performing step 3 of algorithm in two ways : (1) prefetching to overlap the I/O of $S_{j}$ with the ongoingcomputation, and (2) caching to avoid the re-reading of supernodes to be used in the near future. Each processor issues prefetch $\mathrm{I} / \mathrm{O}$ requests based on the schedule generated from the elimination tree and availability of memory space. A static prefetch horizon of two dependency levels per supernode is used, generating at most $2 m$ outstanding read requests on each processor. The prefetch horizon was determined empirically and depends on the per-processor physical memory available for user programs as well as the relative $\mathrm{I} / \mathrm{O}$ and computation rates. $\mathrm{I} / \mathrm{O}$ requests in factor can be both local and non-local; step 3 of Figure 5 requires nonlocal I/O when $S_{j}$ is not stored on local disk. Prefetched non-local data is injected into the communication network when the local computation reaches an appropriate point in the schedule.

\subsection{Results and Analysis}

We ran partitioner and factor on a variety of peer-peer configurations. In all configurations, we used only one local disk at each node. To evaluate the benefit of future

\begin{tabular}{|c|c|c|c|c|}
\hline \multirow{2}{*}{ Matrix } & \multicolumn{2}{|c|}{ Partitioner } & \multicolumn{2}{c|}{ Factorization } \\
\cline { 2 - 5 } & Read & Write & Read & Write \\
\hline skirt & 381 & 403 & 20,200 & 377 \\
sara-1 & 488 & 534 & 49,000 & 509 \\
sara-2 & 301 & 1,939 & 220,800 & 838 \\
\hline
\end{tabular}

Table 7: Application I/O volumes (in MB) for 16 processors.

knowledge provided by the elimination tree, we conducted experiments with two versions of factor-one that used the information for prefetching (factor) and the other that did not (factor-np). In factor-np, processors make explicit $\mathrm{I} / \mathrm{O}$ requests to their peers and service the requests from their peers at particular points in the execution. Specifically, a processor is available to serve $\mathrm{I} / \mathrm{O}$ requests from its peers either after step 4 of the algorithm outlined in Figure 5 or while it is waiting for the completion of its non-local $\mathrm{I} / \mathrm{O}$ requests. As before, all computation on a supernode is performed at the processor in which the data is stored. Since I/O operations are performed on demand, non-local requests pay for one round-trip delay as well as any delay incurred by the processor that is servicing the request. Such delays may occur due to local computation at the service node and due to interactions with other peers.

We used three input matrices in our experiments - skirt, sara- 1 and sara-2. Table 6 presents some characteristics of these matrices. The first two, skirt and sara- 1 correspond to roughly the same number of equations but skirt has fewer non-zeroes and is more sparse. The third matrix, sara-2 is similar in the number of non-zeroes to sara- 1 but corresponds to twice as many equations. As a result, sara-2 is significantly more sparse than the other two, contains relatively thin supernodes and needs more space to store the sparse-matrix data-structures.

Table 7 shows the total amount of I/O performed by both applications for the three matrices. Since the elimination tree is replicated over all processors, the size of partitioner's output, and consequently the size of factor's input, increases with the number of processors. The table shows the $\mathrm{I} / \mathrm{O}$ volumes for 16 processors, the largest possible configuration on our machine.

Table 8 presents the aggregate application-level I/O rates achieved by partitioner. This number is computed by dividing the total volume of $\mathrm{I} / \mathrm{O}$ by the sum of the time spent issuing $\mathrm{I} / \mathrm{O}$ requests and the time spent waiting for them to complete. The superlinear growth in the application-level $\mathrm{I} / \mathrm{O}$ rate, seen for all three phases, is a caching effect. The index file is read in both the Read- 1 and Read- 2 phases. For the 16 processor configuration, the Read-2 phase is operating entirely out of the file cache. Similarly, the write phase benefits from the write-behind nature of the file cache. The performance of writes lags significantly behind that of reads because of a group of small unbuffered writes that dominate the write time. This effect can be easily eliminated by using a buffer to collect these writes (stdio should be adequate).

The breakdown of execution time for partitioner is shown in Table 9. It shows that (1) I/O is a small fraction $(7.6 \%-25 \%)$ of the total execution time and (2) $\mathrm{I} / \mathrm{O}$ scales well with increasing numbers of processors. In fact, $\mathrm{I} / \mathrm{O}$ scales better than the computation for all three matrices. For these matrices, I/O takes less than $25 \%$ of the total program execution time, often significantly less. 


\begin{tabular}{|c|c|c|c|c|c|}
\hline Matrix & $\mathrm{N}$ & $|A|$ & $|L|$ & Type & Description \\
\hline skirt & 45,361 & $1.3 \times 10^{6}$ & $45.8 \times 10^{6}$ & Real & NASA \\
sara-1 & 44,856 & $2.6 \times 10^{6}$ & $30.4 \times 10^{6}$ & Complex & Structural Acoustics \\
sara-2 & 80,651 & $2.9 \times 10^{6}$ & $28.4 \times 10^{6}$ & Complex & Structural Acoustics \\
\hline
\end{tabular}

Table 6: Characteristics of input matrices. N is the number of equations. $|A|$ denotes the number of nonzeros in the input matrix, and $|L|$ is the number of nonzeros in the Cholesky factor.

\begin{tabular}{|c|c|c|c|c|c|c|c|c|c|}
\hline \multirow{2}{*}{ Matrix } & \multicolumn{3}{|c|}{ 4 nodes } & \multicolumn{3}{c|}{ 8 nodes } & \multicolumn{3}{c|}{16 nodes } \\
\cline { 2 - 9 } & Read-1 & Read-2 & Write & Read-1 & Read-2 & Write & Read-1 & Read-2 & Write \\
\hline skirt & 14.1 & 19.4 & 9.9 & 43.0 & 45.8 & 19.7 & 108.8 & 380.9 & 41.1 \\
sara-1 & 12.3 & 15.9 & 9.3 & 18.6 & 29.5 & 18.7 & 106.1 & 430.7 & 15.1 \\
sara-2 & 20.4 & 49.6 & 2.5 & 42.1 & 76.4 & 16.4 & 103.2 & 297.8 & 15.5 \\
\hline
\end{tabular}

Table 8: Aggregate application-level I/O rates (MB/s) for partitioner.

\begin{tabular}{|r|r|r|r|r|r|r|}
\hline \multirow{2}{*}{ Matrix } & \multicolumn{2}{|c|}{4 nodes } & \multicolumn{2}{c|}{8 nodes } & \multicolumn{2}{c|}{16 nodes } \\
\cline { 2 - 7 } & Total & 1/O & Total & \multicolumn{1}{c|}{ //O } & Total & \multicolumn{1}{c|}{ / } \\
\hline skirt & 309.6 & $77.6(25.1 \%)$ & 222.4 & $38.4(17.3 \%)$ & 162.0 & $19.3(11.9 \%)$ \\
sara-1 & 522.3 & $79.2(15.2 \%)$ & 401.7 & $40.1(10.0 \%)$ & 319.8 & $24.3(7.6 \%)$ \\
sara-2 & $2,568.4$ & $541.7(21.1 \%)$ & $2,101.2$ & $319.2(15.2 \%)$ & $1,568.4$ & $177.9(11.3 \%)$ \\
\hline
\end{tabular}

Table 9: Execution time breakdown for partitioner, in seconds

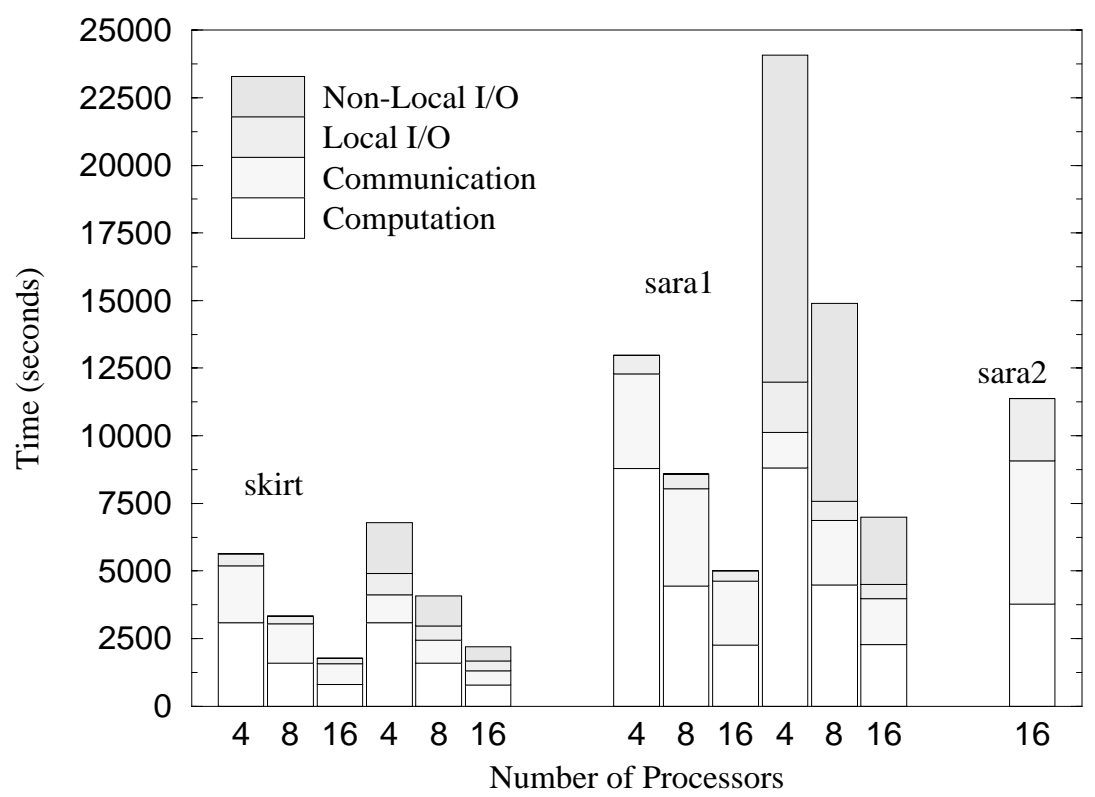

Figure 7: Execution time breakdown for factor and factor-np. The graph shows results for 4-, 8- and 16-processor configurations for both factor and factor-np on skirt and sara-1. For sara-2, only the results for factor on a 16-processor configuration are shown; factoring sara-2 takes too long on other configurations to allow much experimentation. For skirt and sara-1, the first three bars show the results for factor and the second three bars show the results for factor-np. 


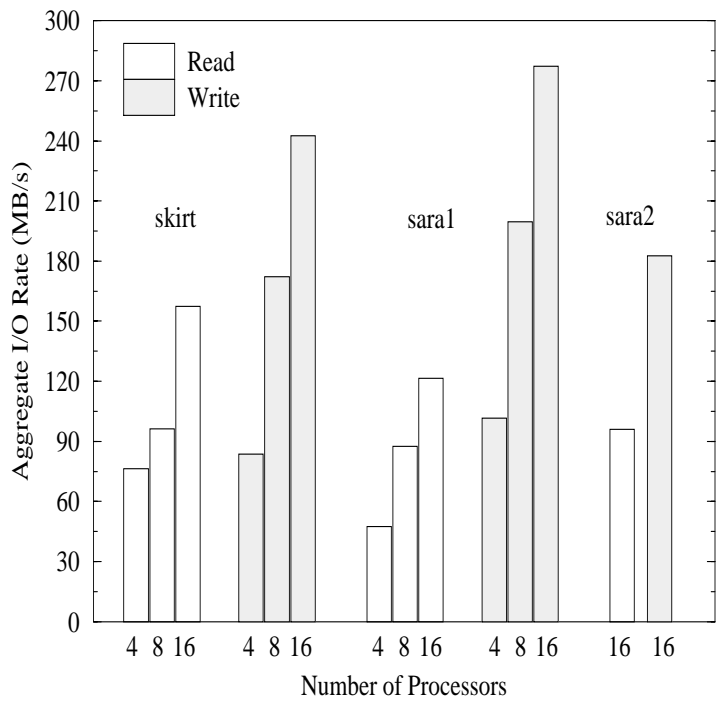

Figure 6: Aggregate application-level I/O rates for factor. Results are shown for 4-, 8- and 16-processor configuration for skirt and sara-1. For sara-2, only the results for the 16processor configuration are shown; experiments for smaller configurations take very long to complete.

Figure 6 shows the aggregate application-level I/O rates seen by factor. It shows that factor is able to achieve an application-level read bandwidth up to $170 \mathrm{MB} / \mathrm{s}$ and an application-level write bandwidth up to $270 \mathrm{MB} / \mathrm{s}$.

The execution time breakdowns for factor and factor-np are shown in Figure 7 . In addition to showing how the different parts of each program scale, this graph also quantitatively demonstrates the utility of knowledge about future $\mathrm{I} / \mathrm{O}$ requests.

The breakdown for factor shows that the computation scales linearly with the number of processors. The I/O performance scales fairly well but not as much as the computation. Communication, however, does not scale well. In all cases, I/O takes a fairly small percentage of the total execution time. Note that the sara-2 input data set was only run on 16 processors, because the program runs for too long on fewer processors. Even for this very sparse matrix, $\mathrm{I} / \mathrm{O}$ takes only about $20 \%$ of the total execution time.

Comparison of these results with corresponding results for factor-np shows that lack of knowledge about future $\mathrm{I} / \mathrm{O}$ requests can degrade performance by between 23 and $86 \%$. Furthermore, the fraction of execution time spent waiting for $\mathrm{I} / \mathrm{O}$ increased from between $1 \%$ and $7 \%$ for factor to between $40 \%$ and $56 \%$ for factor-np. Almost all of the increase in execution time is due to time spent waiting for non-local I/O requests to complete.

The slowdown was more pronounced $(86 \%)$ for sara- 1 than for skirt. We speculate that this difference is due to the different amount of computation performed per supernode. Recall that a processor is available to serve $\mathrm{I} / \mathrm{O}$ requests from other processors either after step 4 of the algorithm outlined in Figure 5 or while it is waiting for the completion of its own non-local I/O requests. The less sparse nature of skirt results in a more uniform distribution of per- supernode computation time. Also since nonzero precision in skirt is less than nonzero precision in sara-1, the average per-supernode computation is also smaller for skirt. In contrast, sara-1 has a large variation in the per-supernode computation times caused by a few very large supernodes. As a result, in certain phases of the computation for sara1 , service of $\mathrm{I} / \mathrm{O}$ requests from other processors is delayed for large intervals causing cascaded performance degradation of non-local I/O. As mentioned above, non-local I/O dominated the $1 / O$ costs for factor-np: for sara-1, $87 \%$ of the I/O time was spent for non-local I/O, constituting $50 \%$ of total runtime.

\section{Lessons Learned}

This section presents the lessons we learned from the studies presented in this paper. We present our experiences as well as guidelines for obtaining high $\mathrm{I} / \mathrm{O}$ performance for $\mathrm{I} / \mathrm{O}$ intensive applications.

\section{Code restructuring is important:}

- For the applications we have studied, it is not difficult to restructure the code to coalesce small requests into much larger ones. Based on our experience with these applications as well as our examination of other NASA satellite-data processing programs, we believe that, while many I/O-intensive applications are currently not designed to generate large $\mathrm{I} / \mathrm{O}$ requests, relatively little effort is required to modify them to do so. In other words, the problem is not that large $\mathrm{I} / \mathrm{O}$ requests cannot be generated, but that programmers have not considered the problem of optimizing their applications to take advantage of the performance benefits provided by larger requests.

- For the applications we have studied, information about future requests was available and could be used to prefetch data. For pathfinder and climate, processors subsample the input files in the partitioning phase. At the end of this phase, every processor has complete information about its future requests for input reads. For the modified version of the out-of-core maxreduction (where modification consisted of a pair of simple loop-splitting and loop-reordering transformations), information about updates to all frequency bands of the output image is known before any updates are performed. For factor and partitioner, the sequence of requests is available from the elimination-tree structure generated by symbolic factorization. Similar experiences have been reported by Patterson et al [15]. They report that after relatively simple loop-splitting transformations, significant knowledge about future I/O requests is available in all five $\mathrm{I} / \mathrm{O}$-intensive programs they studied.

Furthermore, three of the applications in this study (all the ones that write a significant volume of data), could be structured to take good advantage of the writebehind provided by the operating-system file cache.

- For the applications we have studied, it is possible to partition the out-of-core intermediate data so that each processor reads and writes to its own local disk(s). As can be expected, and as we have shown in Section 3 , bandwidths for local disk access are substan- 
tially higher than the bandwidths for non-local accesses. In addition, local accesses are guaranteed not to interfere with $\mathrm{I} / \mathrm{O}$ requests from other processors. This increases the utility of the file cache and makes the overall behavior of the application more predictable. Exploiting locality in this manner is beneficial for outof-core applications $[1,2,14]$ on both client-server and peer-peer configurations. In either configuration, exploiting locality improves $\mathrm{I} / \mathrm{O}$ performance as well as total execution time.

\section{Diskful machines are important:}

Diskful machines (machines with local disks) allow problems to be partitioned such that most of the $\mathrm{I} / \mathrm{O}$ requests are satisfied by local disks. As noted above, local disk accesses have a higher application-level bandwidth with the associated benefit of guaranteed lack of contention for the disk and the file cache. As shown by the results in Sections 4 and 5, local disks attached to compute nodes can help convert programs that request tens to hundreds of gigabytes of $\mathrm{I} / \mathrm{O}$ into compute-bound problems. In combination with code restructuring to exploit locality, diskful machines can improve both the I/O performance and the overall execution time for out-of-core applications.

\section{Complex I/O interfaces are not required:}

- After code restructuring, most requests in the studied applications were large. For large requests, the interface is usually less important.

- Small strided requests were a recurrent pattern in the original versions of pathfinder and climate. Nestedstrided requests [12] have been proposed for just such patterns. However we found that these patterns were caused by the embedding of small I/O requests in the innermost loops. Relatively straightforward loop restructuring, including loop splitting, interchanging the order of nested loops [18] and fusing multiple requests were sufficient to coalesce these requests into large block I/O requests.

- None of the applications studied required collective $\mathrm{I} / \mathrm{O}[1,3,16]$. This is not surprising given the size of the requests after code restructuring. All of the applications are parallelized in SPMD fashion. In our earth-science applications all processes are independent (apart from initial and possibly final synchronization). Independent $\mathrm{I} / \mathrm{O}$ requests were able to utilize the servers when they would have been idle in a collective- $\mathrm{I} / \mathrm{O}$ model (see Section 4).

We recognize that this paper describes experiences with only four programs. However, we believe that a substantial class of $\mathrm{I} / \mathrm{O}$-intensive programs will be able to achieve good $\mathrm{I} / \mathrm{O}$ performance with simple $\mathrm{I} / \mathrm{O}$ interfaces. This belief is based on our examination of other NASA satellite-data processing programs and on the experiences reported by Patterson et al [15]. The characterization study by Crandall et al [4] provides another example. It describes a significantly different program running on a machine with much lower I/O bandwidth (the JPL terrain rendering application running on an Intel Paragon) that is able to achieve relatively good $\mathrm{I} / \mathrm{O}$ performance with just asynchronous $\mathrm{I} / \mathrm{O}$ requests.
We speculate that with sufficient $\mathrm{I} / \mathrm{O}$ bandwidth and efficient asynchronous $\mathrm{I} / \mathrm{O}$ support and an interface similar to lio_listio(), most I/O-intensive programs will be able to achieve good $\mathrm{I} / \mathrm{O}$ performance.

\section{Good performance on peer-peer systems is possible:}

Our experience with applications that do substantial I/O and computation on peer-peer configurations was mixed. On one hand, the performance of pathfinder on peer-peer configurations was poor; on the other hand, factor achieved excellent performance. The problem of achieving good computation performance on processors that are serving data to others has been previously noted by Kotz and Cai [8]. In their experiments on a cluster of RS6000s, they found that serving off-processor $\mathrm{I} / \mathrm{O}$ requests can slow a relatively simple parallel program by between $17 \%$ and $98 \%$. We believe that knowledge of future $\mathrm{I} / \mathrm{O}$ requests (local and offprocessor) is likely to be the key to achieving good I/O performance on peer-peer configurations. Our experiments with pathf inder used a general-purpose parallel-I/O library which served requests as they arrived and had no information about future $\mathrm{I} / \mathrm{O}$ requests. In contrast, the $\mathrm{I} / \mathrm{O}$ module in factor had access to extensive information about future requests and was able to control the scheduling of $\mathrm{I} / \mathrm{O}$ requests. The other version of factor, which did not take advantage of this knowledge, performed significantly worse.

\section{Conclusions}

In this paper we have shown that $\mathrm{I} / \mathrm{O}$-intensive parallel applications can be optimized so that $\mathrm{I} / \mathrm{O}$ is not the limiting factor in their performance. The results from both microbenchmarks and complete applications, run on an IBM SP-2 with multiple disks per node, show that we can achieve high $\mathrm{I} / \mathrm{O}$ rates from the hardware and into the applications. We have been able to convert programs with very large $\mathrm{I} / \mathrm{O}$ requirements whose performance appears to be limited by the I/O capabilities of the parallel machine into compute-bound programs. Our experience has shown that achieving high $\mathrm{I} / \mathrm{O}$ performance does not require complex $\mathrm{I} / \mathrm{O}$ strategies; rather, appropriate restructuring of the applications to use local secondary storage for staging intermediate results and producing relatively small numbers of large I/O requests allows an $\mathrm{I} / \mathrm{O}$ library or the vendor filesystem to provide a high $\mathrm{I} / \mathrm{O}$ bandwidth to the application. In addition, overlapping the $\mathrm{I} / \mathrm{O}$ with computation, either in the application or in the operating system, provided large performance benefits. For the applications we have studied, this benefit derives from the out-of-core nature of the algorithms used, which are required because of the extremely large data sets to be processed. These out-of-core algorithms did not require complex I/O interfaces to achieve high $\mathrm{I} / \mathrm{O}$ bandwidths. A relatively simple interface like POSIX 1 io_listio() was adequate as long as the application and $\mathrm{I} / \mathrm{O}$ system were configured properly.

\section{Acknowledgements}

We would like to thank our shepherd, David Kotz, for his detailed comments. We would like to thank Tonjua Hines and Steve Kempler from the Earth Science Data \& Information Systems Project (ESDIS) at NASA Goddard Space 
Flight Center for many invaluable discussions about NASA's remote sensing data processing requirements, and for helping us gain access to NASA programs. We would also like to thank Peter Smith and Mary James of the Goddard Distributed Active Archive Center (DAAC) for providing the pathfinder and climate programs.

\section{References}

[1] R. Bennett, K. Bryant, A. Sussman, R. Das, and J. Saltz. Jovian: A framework for optimizing parallel I/O. In Proceedings of the 1994 Scalable Parallel Libraries Conference, pages 10-20. IEEE Computer Society Press, October 1994.

[2] R. Bordawekar, A. Choudhary, K. Kennedy, C. Koelbel, and M. Paleczny. A model and compilation strategy for out-of-core data parallel programs. In Proceedings of the Fifth ACM SIGPLAN Symposium on Principles 8 Practice of Parallel Programming, pages 1-10. ACM Press, July 1995. ACM SIGPLAN Notices, Vol. 30, No. 8.

[3] A. Choudhary, R. Bordawekar, M. Harry, R. Krishnaiyer, R. Ponnusamy, T. Singh, and Rajeev Thakur. PASSION: Parallel and scalable software for InputOutput. Technical Report SCCS-636, NPAC, September 1994. Also available as CRPC Report CRPCTR94483.

[4] P. Crandall, R. Aydt, A. Chien, and D. Reed. Input/output characteristics of scalable parallel applications. In Proceedings Supercomputing'95, December 1995.

[5] J.J. Dongarra, J. DuCroz, I.S.Duff, and S. Hammarling. A set of level 3 Basic Linear Algebra Subprograms. ACM Transactions on Mathematical Software, 16(1):1-17, 1990.

[6] M. King. Earth Observation System Project Science homepage.

http://spso.gsfc.nasa.gov/spso_homepage.html, 1995.

[7] D. Kotz. Disk-directed I/O for MIMD multiprocessors. In Proceedings of the 1994 Symposium on Operating Systems Design and Implementation, pages 61-74. ACM Press, November 1994.

[8] D. Kotz and T. Cai. Exploring the use of I/O nodes for computation in a MIMD processor. In Proceedings of the IPPS'95 Third Annual Workshop on Input/Output in Parallel and Distributed Systems, pages 78-89, April 1995.

[9] D. Kotz and N. Nieuwejaar. File-system workload on a scientific multiprocessor. IEEE Parallel \&\& Distributed Technology, 3(1):51-60, Spring 1995.

[10] J. W. H. Liu. The Role of Elimination Trees in Sparse Factorization. SIAM Journal of Matrix Analysis and Applications, (11):134-172, 1990.

[11] E. G. Ng and B. W. Peyton. Block Sparse Cholesky Algorithms on Advanced Uniprocessor Computers. SIAM Journal on Scientific Computing, 14(5):10341056, September 1993.
[12] N. Nieuwejaar and D. Kotz. Low-level interfaces for high-level parallel I/O. In Proceedings of the IPPS'95 Third Annual Workshop on Input/Output in Parallel and Distributed Systems, pages 47-62, April 1995.

[13] W. Norcutt. IOZONE benchmark program. Available at ftp:/ftp.cs.umn.edu/packages/FreeBSD/2.0.5RELEASE/ports/utils/iozone, 1991.

[14] M. Paleczny, K. Kennedy, and C. Koelbel. Compiler support for out-of-core arrays on parallel machines. In Proceedings of the Fifth Symposium on the Frontiers of Massively Parallel Computation, pages 110-118. IEEE Computer Society Press, February 1995.

[15] R. Patterson, G. Gibson, E. Ginting, D. Stodolsky, and J. Zelenka. Informed prefetching and caching. In Proceedings of the Fifteenth ACM Symposium on Operating System Principles, pages 79-95, December 1995.

[16] J. Rosario and A. Choudhary. High-performance I/O for massively parallel computers: Problems and prospects. IEEE Computer, 27(3):59-68, March 1994.

[17] Edward Rothberg and Anoop Gupta. An Efficient Block-Oriented Approach to Parallel Sparse Cholesky Factorization. In Proceedings of Supercomputing '93, pages 503-512, Portland, OR, November 1993.

[18] M. Wolfe. High Performance Compilers for Parallel Computing. Addison-Wesley, 1995. 